\title{
How Use Cases Drove the Design of the HELICS Co-simulation Framework
}

\section{Bryan Palmintier, PhD}

Senior Research Engineer

Power Systems Engineering Center National Renewable Energy Laboratory

HELICS Mini-Tutorial

March 16, 2018
INREL LL Lawrence Livemore Argonne

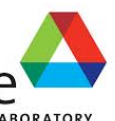

Northwest

Pacific Northwest
NATIONAL LABORATO 


\section{The Vision for HELICS}

\section{HELICS}

- A Co-simulation platform that meets the current and future needs of the power system community

Transmission

Distribution

Communications

Markets

Controls

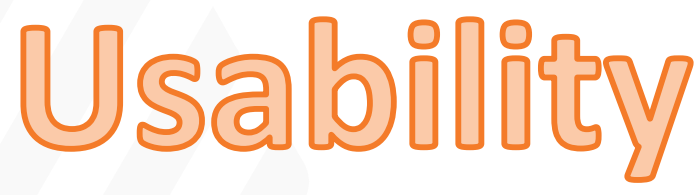

Others

- Scalability

We must scale to large number of different simulations

We must operate at multiple machine scales

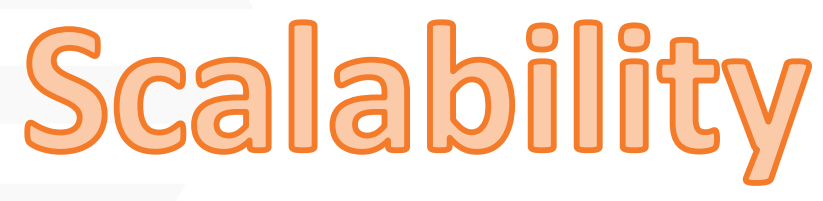




\section{The Vision for HELICS}

\section{HELICS}

- A Co-simulation platform that meets the current and future needs of the power system community

Transmission

Distribution

Communications

Markets

Controls

Others

- Scalar

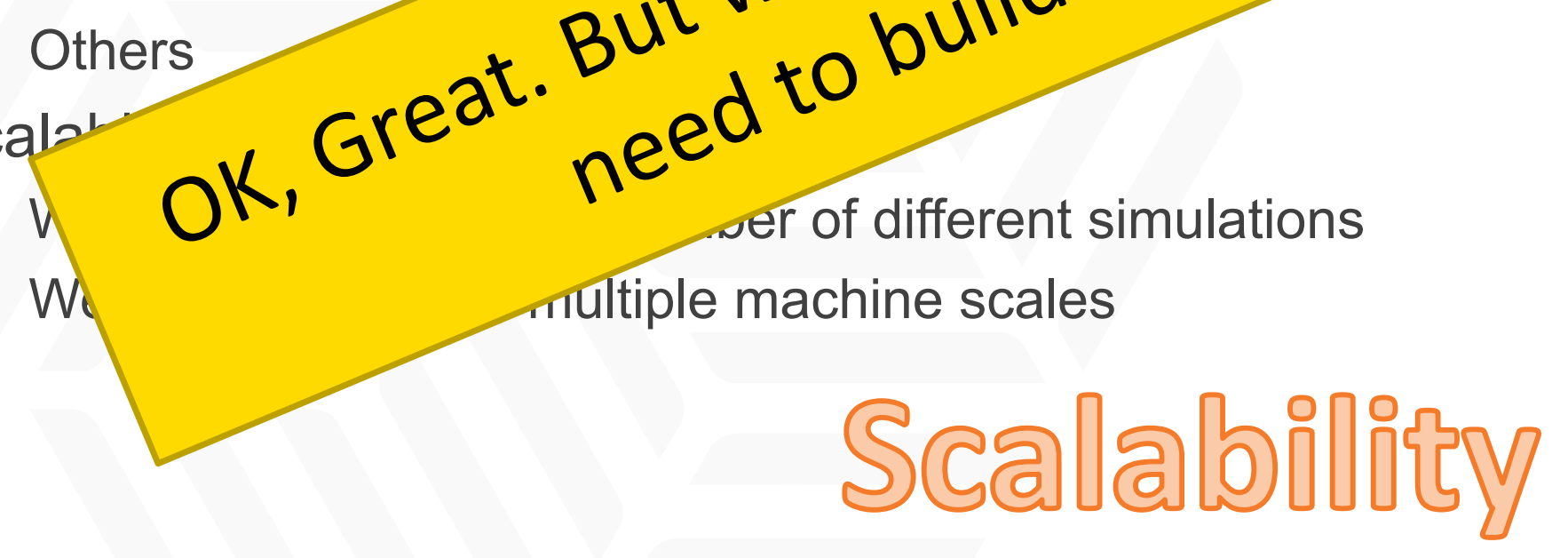




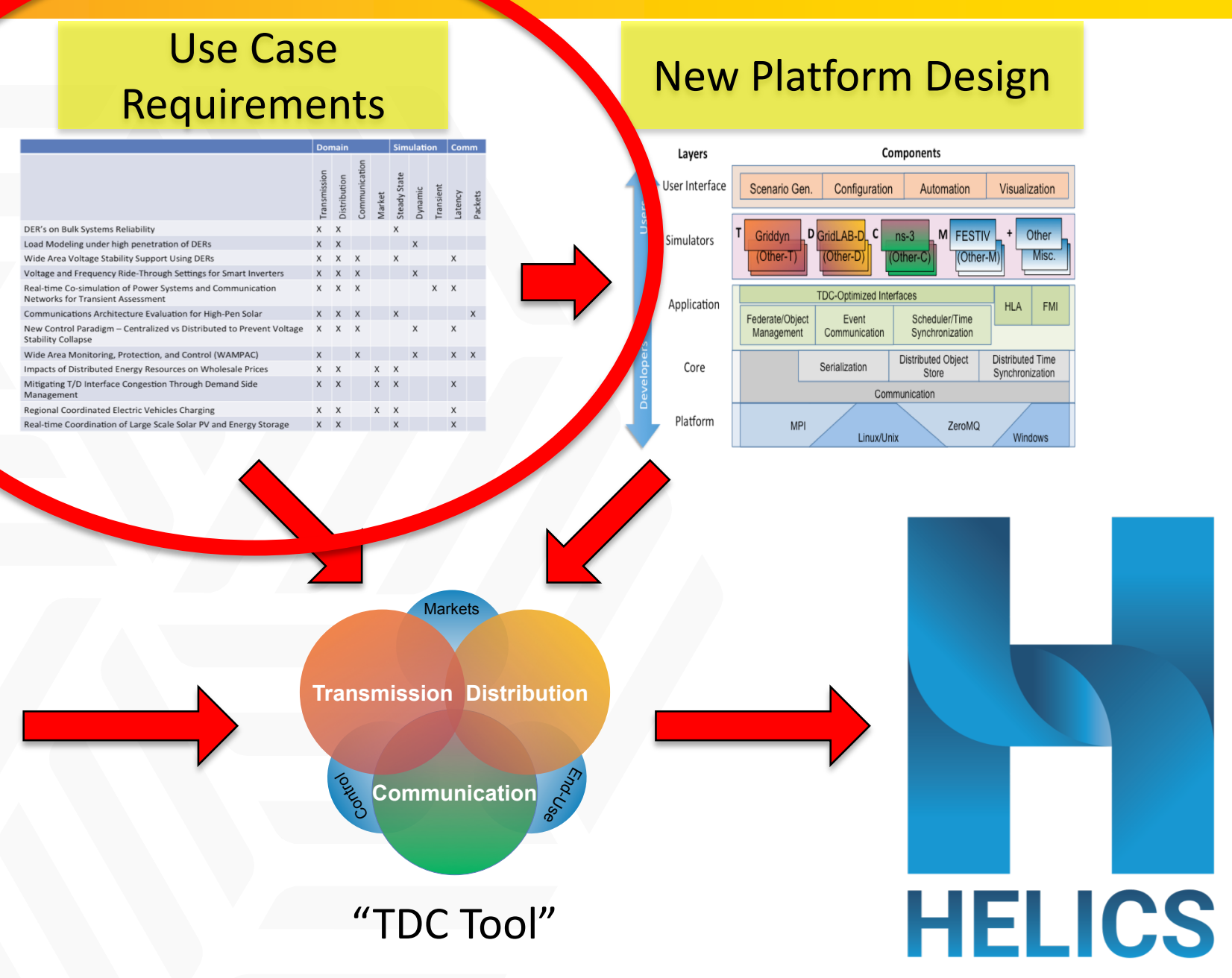




\section{Original 12 Use Cases}

1. Impacts of DER's on Bulk Systems Reliability

2. Impacts of Distributed Energy Resources on Wholesale Prices

3. Regional Coordinated Electric Vehicles Charging

4. Real-time Coordination of Large-Scale Solar PV and Energy Storage

5. Evaluate modeling adequacy of composite load model under high penetration of DERs

6. Mitigating Transmission-Distribution Interface Congestion Through Demand Side Management

7. New Control Paradigm - Centralized vs Distributed to Prevent Voltage Stability Collapse

8. Wide Area Monitoring, Protection, and Control (WAMPAC)

9. Real-time Co-simulation of Power Systems and Communication Networks for Transient Assessment

10. Communications Architecture Evaluation for High-Pen Solar

11. Adaptive Voltage and Frequency Ride-Through Settings for Smart Inverters

12. Wide Area Voltage Stability Support Using DERs 


\section{Original 12 Use Cases - T}

1. Impacts of DER's on Bulk Systems Reliability

2. Impacts of Distributed Energy Resources on Wholesale Prices

3. Regional Coordinated Electric Vehicles Charging

4. Real-time Coordination of Large-Scale Solar PV and Energy Storage

5. Evaluate modeling adequacy of composite load model under high penetration of DERs

6. Mitigating Transmission-Distribution Interface Congestion Through Demand Side Management

7. New Control Paradigm - Centralized vs Distributed to Prevent Voltage Stability Collapse

8. Wide Area Monitoring, Protection, and Control (WAMPAC)

9. Real-time Co-simulation of Power Systems and Communication Networks for Transient Assessment

10. Communications Architecture Evaluation for High-Pen Solar

11. Adaptive Voltage and Frequency Ride-Through Settings for Smart Inverters

12. Wide Area Voltage Stability Support Using DERs 


\section{Original 12 Use Cases - D}

1. Impacts of DER's on Bulk Systems Reliability

2. Impacts of Distributed Energy Resources on Wholesale Prices

3. Regional Coordinated Electric Vehicles Charging

4. Real-time Coordination of Large-Scale Solar PV and Energy Storage

5. Evaluate modeling adequacy of composite load model under high penetration of DERs

6. Mitigating Transmission-Distribution Interface Congestion Through Demand Side Management

7. New Control Paradigm - Centralized vs Distributed to Prevent Voltage Stability Collapse

8. Wide Area Monitoring, Protection, and Control (WAMPAC)

9. Real-time Co-simulation of Power Systems and Communication Networks for Transient Assessment

10. Communications Architecture Evaluation for High-Pen Solar

11. Adaptive Voltage and Frequency Ride-Through Settings for Smart Inverters

12. Wide Area Voltage Stability Support Using DERs 


\section{Original 12 Use Cases - C}

1. Impacts of DER's on Bulk Systems Reliability

2. Impacts of Distributed Energy Resources on Wholesale Prices

3. Regional Coordinated Electric Vehicles Charging

4. Real-time Coordination of Large-Scale Solar PV and Energy Storage

5. Evaluate modeling adequacy of composite load model under high penetration of DERs

6. Mitigating Transmission-Distribution Interface Congestion Through Demand Side Management

7. New Control Paradigm - Centralized vs Distributed to Prevent Voltage Stability Collapse

8. Wide Area Monitoring, Protection, and Control (WAMPAC)

9. Real-time Co-simulation of Power Systems and Communication Networks for Transient Assessment

10. Communications Architecture Evaluation for High-Pen Solar

11. Adaptive Voltage and Frequency Ride-Through Settings for Smart Inverters

12. Wide Area Voltage Stability Support Using DERs 


\section{Original 12 Use Cases - \\ Many Time Scales}

1. Impacts of DER's on Bulk Systems Reliability

2. Impacts of Distributed Energy Resources on Wholesale Prices

3. Regional Coordinated Electric Vehicles Charging

4. Real-time Coordination of Large-Scale Solar PV and Energy Storage

5. Evaluate modeling adequacy of composite load model under high penetration of DERs

6. Mitigating Transmission-Distribution Interface Congestion Through Demand Side Management

7. New Control Paradigm - Centralized vs Distributed to Prevent Voltage Stability Collapse

8. Wide Area Monitoring, Protection, and Control (WAMPAC)

9. Real-time Co-simulation of Power Systems and Communication Networks for Transient Assessment

10. Communications Architecture Evaluation for High-Pen Solar

11. Adaptive Voltage and Frequency Ride-Through Settings for Smart Inverters

12. Wide Area Voltage Stability Support Using DERs 


\section{HELICS}

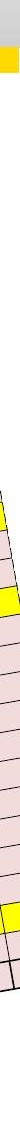




\section{Detailed use case analysis}

\section{HELICS}

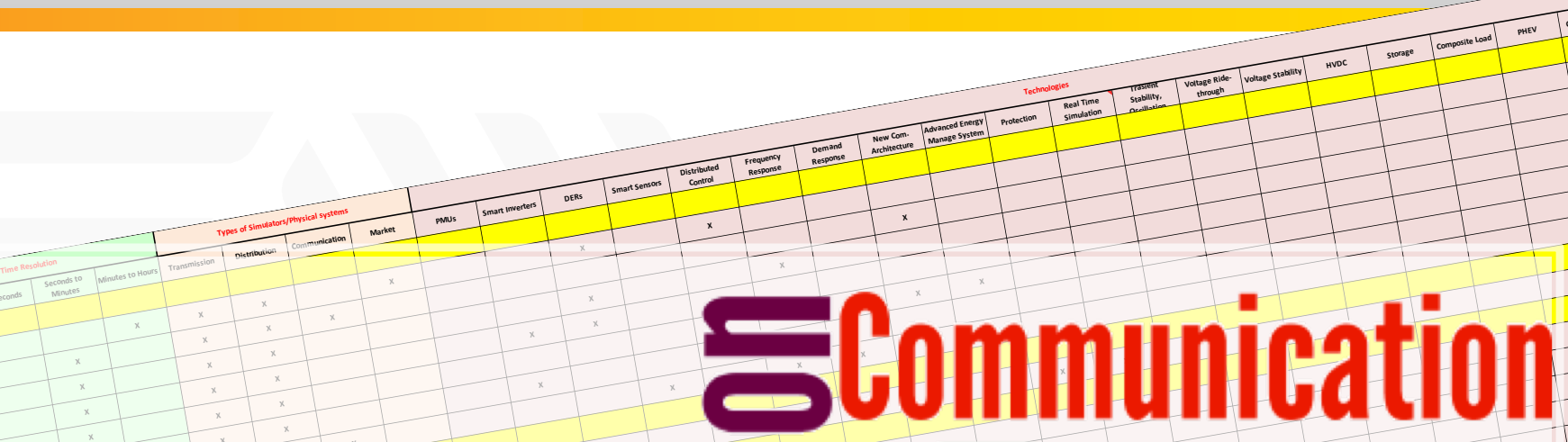

Ride-throught Oscillation

SMa

is on

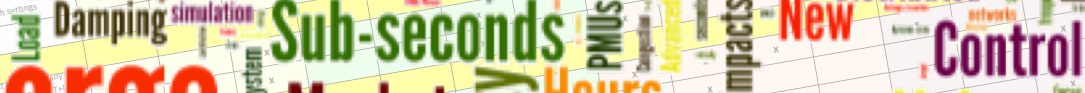

2010 Market

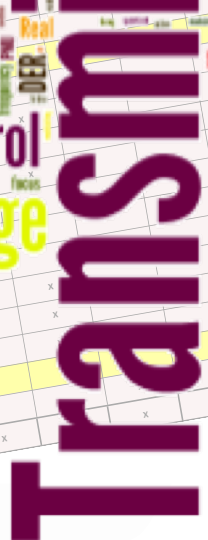

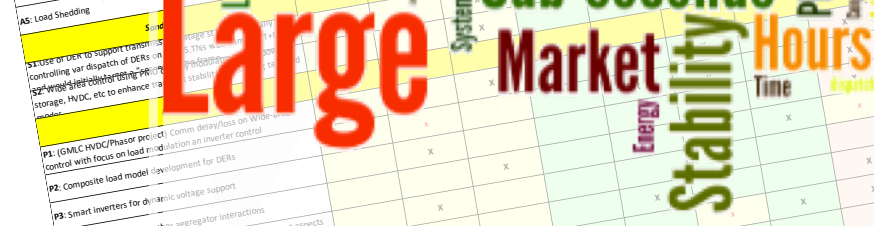

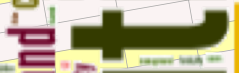

Response

要
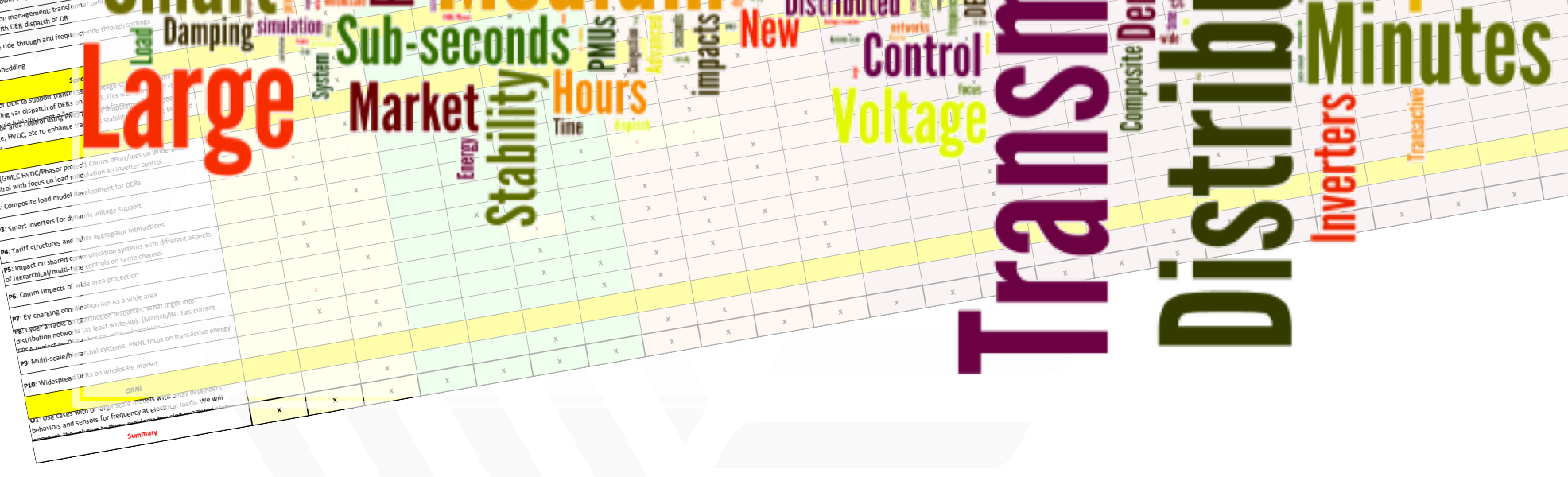


\section{Tool Development Workshop}

- Held a small workshop at NREL to determine software development needs.

- Used the use case descriptions to derive key features necessary for a TD\&C cosimulation tool.

Distilled into "must have" features, and "would be nice" features.

- Evaluated existing toolsets against these requirements.

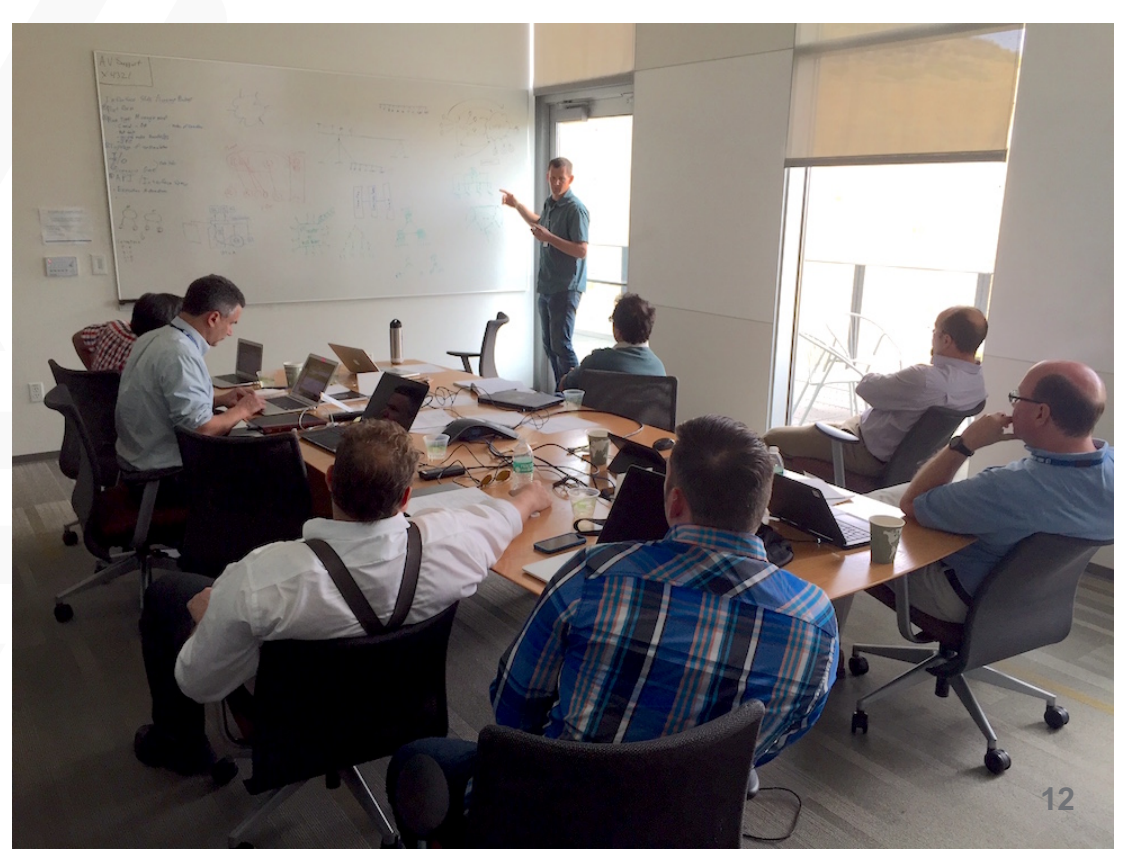




\section{Summary Table}

DER's on Bulk Systems Reliability

Load Modeling under high penetration of DERs

Wide Area Voltage Stability Support Using DERs

Voltage and Frequency Ride-Through Settings for Smart Inverters

Real-time Co-simulation of Power Systems and Communication

Networks for Transient Assessment

Communications Architecture Evaluation for High-Pen Solar

New Control Paradigm - Centralized vs Distributed to Prevent Voltage

Stability Collapse

Wide Area Monitoring, Protection, and Control (WAMPAC)

Impacts of Distributed Energy Resources on Wholesale Prices

Mitigating T/D Interface Congestion Through Demand Side

Management

Regional Coordinated Electric Vehicles Charging

Real-time Coordination of Large Scale Solar PV and Energy Storage

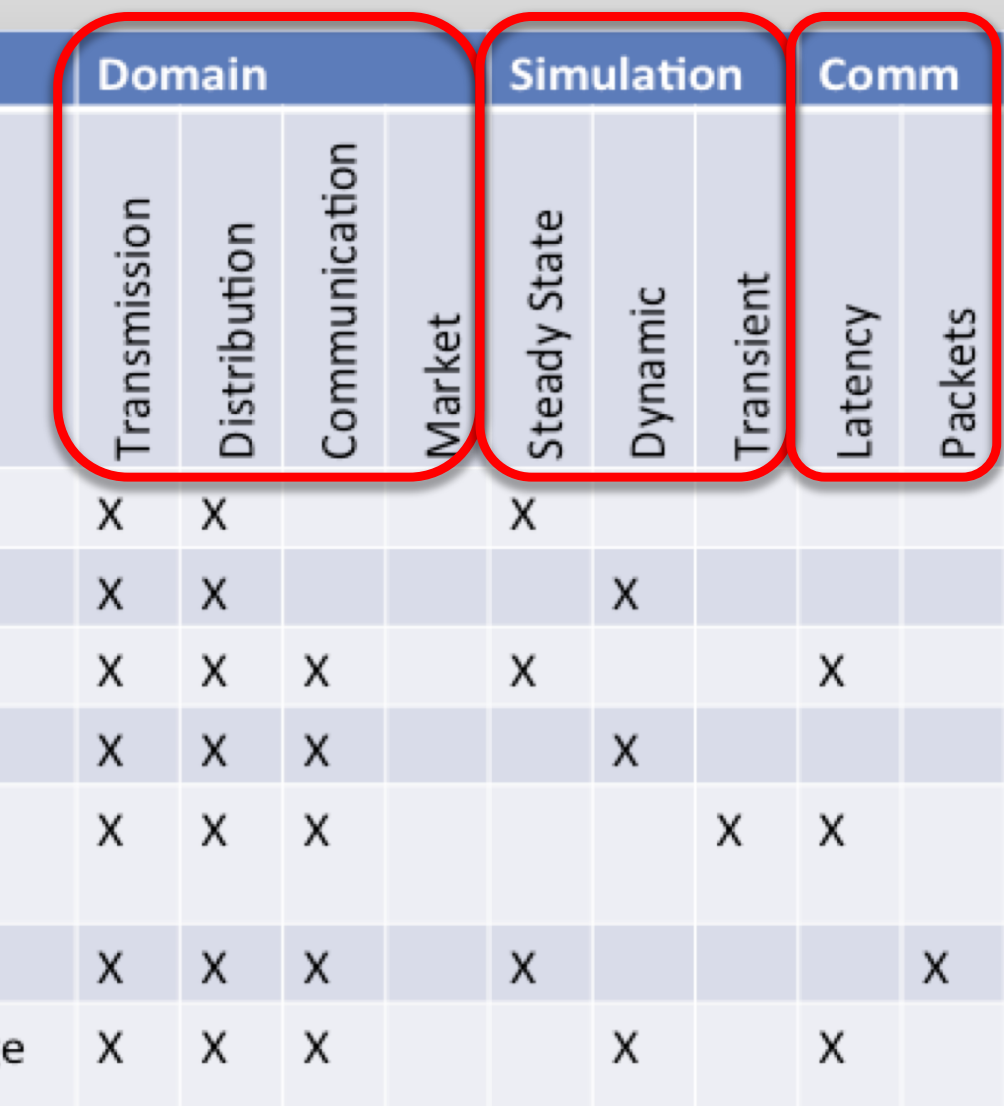

\begin{tabular}{|l|l|l|l|l|l|l|l|l|}
\hline & & $x$ & & & $x$ & $x$ & $x$ \\
\hline$x$ & $x$ & & $x$ & $x$ & & & \\
\hline$x$ & $x$ & & $x$ & $x$ & & $x$
\end{tabular}

\begin{tabular}{ccc|c|c|cc}
$X$ & $X$ & & $X$ & & $X$ & 13 \\
\hline$X$ & $X$ & & & $X$ & & $X$
\end{tabular}


- Tool/platform released open source with (nearly) all use cases accessible with the open-source tool

- Support variety of simulation types

- Discrete Event

- Time Series

- Quasi-Static Time Series

- Phasor (Dynamics)

- "Easily" incorporate existing tools/elements

- From other labs

- From vendors and other developers

- Make provisions for open-source \& commercial software

Intended stakeholders:

- National Labs

- Academics/Students

- Utility Planners

- Vendors

- Consultants 
- Support massive problem scales

- 2 - 100,000 federates

- For example, simultaneously simulate all T, D, and C for the State of California

- Support a wide variety of compute systems

- Laptop/Workstation (Windows and Mac)

- High-performance computing, including cloud/servers

- Focus on usability (research-grade usability)

- Standard file structures and I/O

- Execution tools/scripts

- Documentation and Examples 


\section{HELICS Layered Design}

\section{HELICS}

Layers

\section{\begin{tabular}{|l|l|l|l|l}
\hline User Interface & Scenario Gen. & Configuration & Automation & Visualization
\end{tabular}}

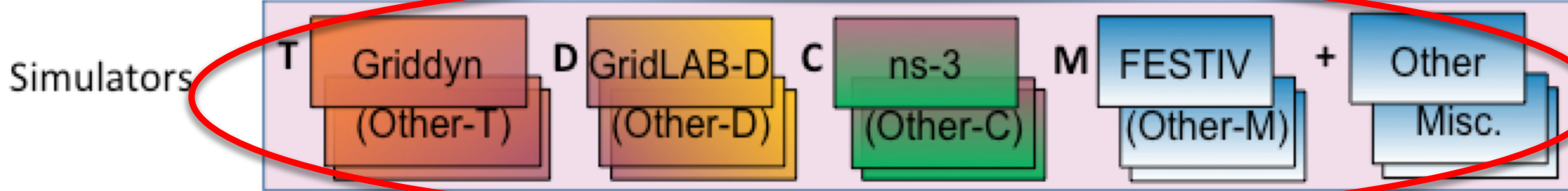

Application

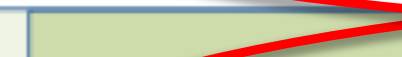

TDC-Optimized Interfaces

\section{Federate/Object} Management
Event

Communication

Scheduler/Time

Synchronization

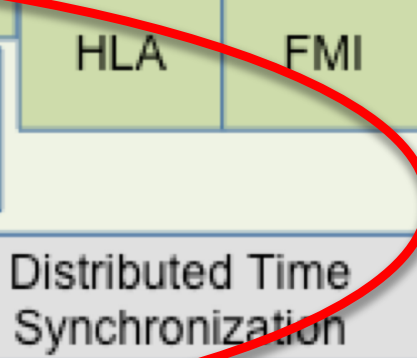
Store

\section{Communication}

Platform

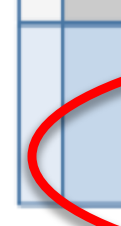

MPI

\section{Linux/Unix}

ZeroMQ 


\section{Types of Federates}

1. Value: Nominally physical value

E.g. Voltage, Current, Power, etc.

Support for co-iteration

2. Message: Nominally data (only) sent over communication system

E.g. Control signals, sensors, market signals

Connectivity only (see below)

Point to point or broadcast

3. Filters: Manipulate data in transit, e.g. ICT simulation

E.g. Delay, ossy channel, Full packet simulation (ns-3)

Rerouting for complex ICT in federates

Allows swappable fidelity for communication simulation

- For all: Consistent API across platform:

Single executable, Zero-MQ, MPI

Expose both discrete event and time series (DE under hood) 


\section{HELICS ${ }^{\mathrm{TM}}$ : Hierarchical Engine for Large-scale Infrastructure Simulation}

\section{HELICS}

Requirements:

- Scalable: 2-100,000+ Federates

- Cross-platform: HPC (Linux), Cloud, Workstations, Laptops (Windows/OSX)

- Modular: mix and match tools

- Minimally invasive: easy to use lab/commercial/open tools

- Open Source: BSD-style

- Many Simulation Types:

- Discrete Event

- QSTS

- Dynamics

- Co-iteration enabled: "tight coupling"

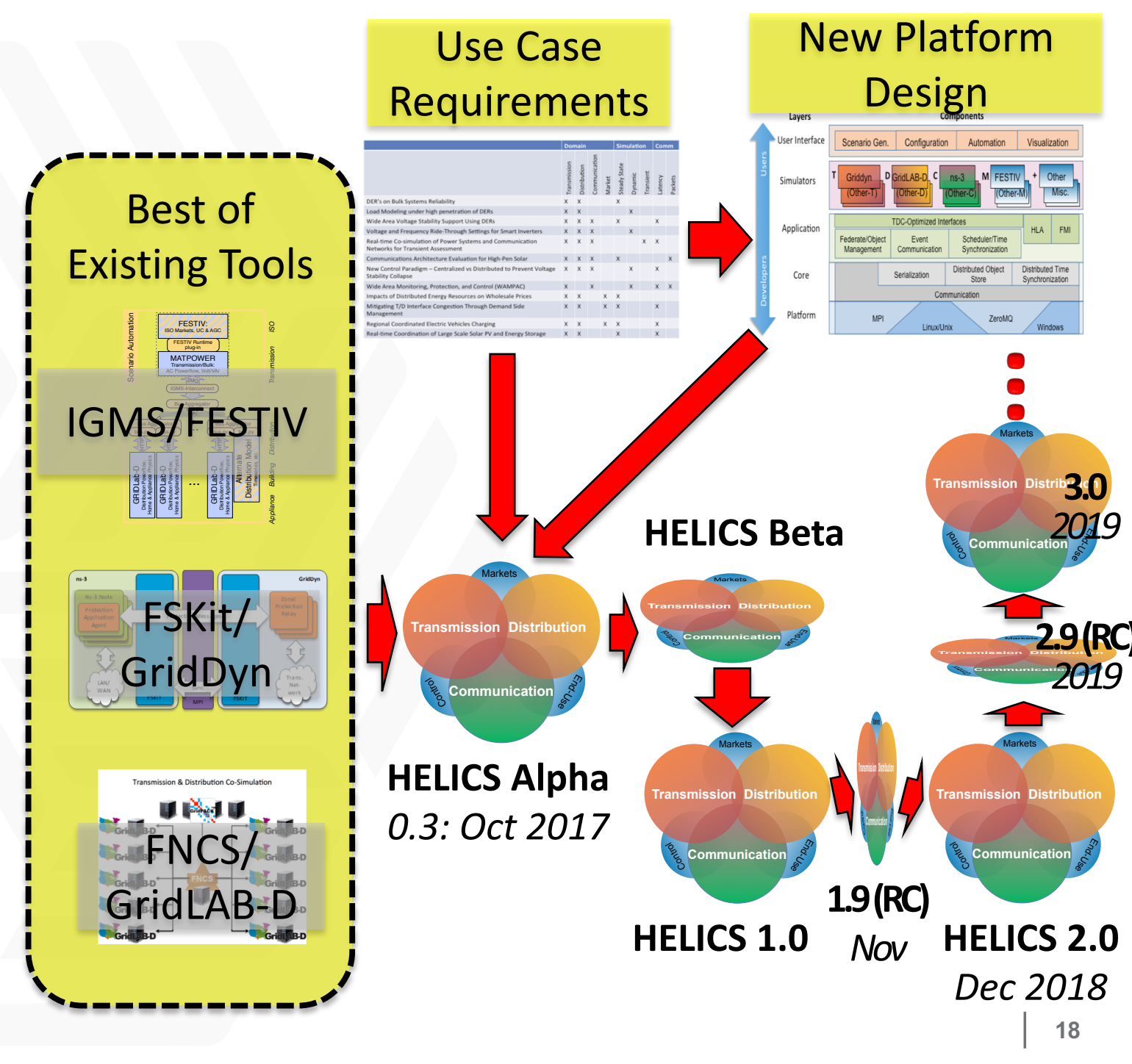




\section{Thank you!}

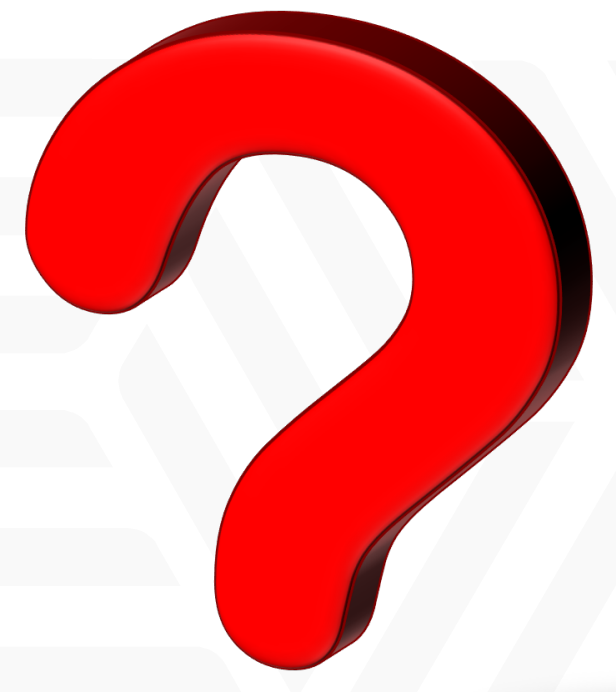

Further Reading: B. Palmintier, D. Krishnamurthy, P. Top, S. Smith, J. Daily, and J. Fuller, "Design of the HELICS High-Performance TransmissionDistribution-Communication-Market Co-Simulation Framework," in Proc. of the 2017 Workshop on Modeling and Simulation of Cyber-Physical Energy Systems, Pittsburgh, PA, 2017.

\section{Bryan.Palmintier@nrel.gov}

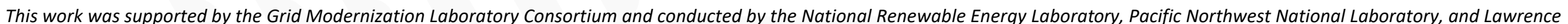

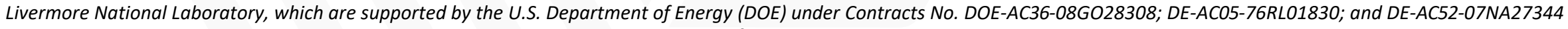

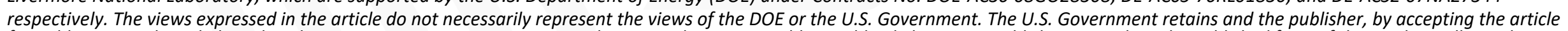

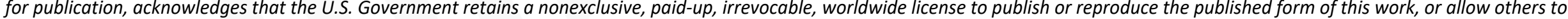
do so, for U.S. Government purposes. 